\title{
Photodynamics of clusters of the major components of the atmosphere
}

\author{
V. Aquilanti, ${ }^{1}$ M. Bartolomei, ${ }^{1}$ D. Cappelletti, ${ }^{2}$ E. Carmona-Novillo, ${ }^{1}$ and F. Pirani ${ }^{1}$ \\ ${ }^{1}$ Dipartimento di Chimica dell' Università di Perugia, Via Elce di Sotto 8, 06123 Perugia, Italia \\ ${ }^{2}$ Dipartimento di Ingegneria Civile e Ambientale dell' Università di Perugia, Via Duranti 1, 06125 Perugia, Italy
}

\begin{abstract}
Weakly interacting molecules leading to collisional complexes, and to either stable or metastable dimers, potentially play an important role in molecular and surface physics, in astrophysics, in atmospheric photochemistry and physics, and climate. Accurate intermolecular potential energy surfaces for the major components of the atmosphere, leading to the characterization of the $\mathrm{O}_{2}-\mathrm{O}_{2}, \mathrm{~N}_{2}-\mathrm{N}_{2}$ and $\mathrm{N}_{2}-\mathrm{O}_{2}$ dimers, have been obtained from the analysis of scattering experiments from our laboratory, also exploiting where available second virial coefficient data. A spherical harmonic expansion functional form describes the geometries of the dimers and accounts for the relative contributions to the intermolecular interaction from components of different nature. For $\mathrm{O}_{2}-\mathrm{O}_{2}$, singlet, triplet and quintet surfaces are obtained accounting for the role of spin-spin coupling. The new surfaces allow the full characterization of structure and internal dynamics of the clusters, whose bound states and eigenfunctions are obtained by exact quantum mechanics. Besides the information on the nature of the bond, these results can be of use in modelling the role of dimers in air and the calculated rotovibrational levels provide a guidance for the analysis of spectra, thus establishing the ground for atmospheric monitorings. Reference is also briefly made to recent insight on the role of water.
\end{abstract}

\section{INTRODUCTION}

An important goal of atmospheric research is the assess the role of weakly interacting species with the aim of quantifying the impact of their optical and dynamical properties in key issues like climate change, radiation budget and global warming. We present here an account on recent progress of our understanding of the dimers of the major components of the atmosphere. Potential energy surfaces (PES) have been characterized by scattering experiments for the $\mathrm{O}_{2}-\mathrm{O}_{2}$ [1] and $\mathrm{N}_{2}-\mathrm{N}_{2}$ [2] cases. Quantum mechanical predictions of spectral features have been preliminary reported [3], other papers having published on the ground PES $\mathrm{N}_{2}-\mathrm{N}_{2}$ [2] and $\mathrm{O}_{2}-\mathrm{O}_{2}$ [4] and recently also on $\mathrm{N}_{2}-\mathrm{O}_{2}$ [5]. Another paper deals with additional excited PES for these systems [6]. An interesting feature is that configurations corresponding to minima in the PES differ in the different cases.

In recent years experimental and theoretical interest has been focused on the study of weakly bound complexes, and we consider here both those regarding the major components present in the atmosphere and other species relevant for its modeling. High resolution spectra for van der Waals clusters can give insight into the nature of intermolecular forces and of internal dynamics, but results for the case of non polar molecules are limited. For them, the interactions involve dispersion forces (induced multipole-induced multipole) which usually are weaker than those arising from electrostatic effects (permanent multipole-permanent multipole), and their complexes rarely have transition moments active in the medium infra-red or microwave ranges. As a consequence, experimental information on the equilibrium geometry and on the bond energy even for diatom-diatom complexes is scarce and a full characterization of the interaction is often lacking. Therefore, new experiments are necessary and a simultaneous analysis of different experimental data is recommended. In our work, interferences effects in scattering cross sections are measured, obtaining information on interaction potentials, which in the case of anisotropic intermolecular potentials require advanced molecular beam techniques, and possibly a control of mutual orientation.

Our current knowledge on the structure of the dimers is presented in the next section. A further section deals with their dynamics and is followed by concluding remarks.

\section{DESCRIPTION OF THE INTERACTION}

We give some background information on the interactions and their representations. From spectroscopic studies available for $\left(\mathrm{N}_{2}\right)_{2}$, the infrared spectrum [7] suggested that the equilibrium conformation is T-shaped with the center-of-mass of the monomers approximately separated by $3.7-4.2 \AA$. The $\left(\mathrm{N}_{2}\right)_{2}$ dimer has been studied by ab-initio methods which have provided several alternatives for the interaction potentials [8-15], yielding different results for the equilibrium geometry and for the bond energy of the ground state. The latest $a b$-initio methods yielded as most stable geometries a $\mathbf{T}$-shaped and a $\mathbf{Z}$ one (see Figure 1) with bond energy in the range $70-80 \mathrm{~cm}^{-1}[11,12]$, a frequency of the stretching van der Waals mode of $22 \mathrm{~cm}^{-1}$ 
and internal rotation barriers with a maximum at $30 \mathrm{~cm}^{-1}$, in fair agreement with experimental results of Long et al. [7]. Spectroscopic and theoretical information on the $\mathrm{N}_{2}-\mathrm{O}_{2}$ system is very scarce, see e.g. [16].

Another experimental source of information is the second virial coefficient. Low temperature data depend on the full anisotropic potential energy surface while at high temperature only the spherical part of the interaction is of relevance. Data are available for $\mathrm{O}_{2}-\mathrm{O}_{2}$ in a wide temperature range and accurate results have been recently derived for the $\mathrm{N}_{2}-\mathrm{N}_{2}$ system from new acoustic measurements [17]. For the $\mathrm{N}_{2}-\mathrm{O}_{2}$ system, for which information is limited, the main features can be indirectly derived as average of those for $\mathrm{O}_{2}-\mathrm{O}_{2}$ and $\mathrm{N}_{2}-\mathrm{N}_{2}$, being the correction due to the "excess" of the second virial coefficient for mixtures negligible, exploiting the similarity of the nature of the interaction in $\mathrm{N}_{2}-\mathrm{N}_{2}$, and $\mathrm{O}_{2}-\mathrm{O}_{2}[18,19]$.

The measurement of quantum mechanical interference effects in the scattering cross-sections provides data which, together with accurate second virial coefficients, yields information on the intermolecular potential and on the dimer structure. We have demonstrated the use of this technique for the $\mathrm{O}_{2}-\mathrm{O}_{2}$ case [1], for which not only the singlet, but also the triplet and quintet potential energy surfaces have been characterized. In the velocity dependence of the integral cross-section, the "glory" oscillations, overimposed to a smooth average component, are a probe of the depth of the potential well and of its position, while the absolute value of the cross-section depends on the long range attraction. In our laboratory, experiments can be performed by using "hot" molecules $\left(T_{\text {rot }} \simeq 10^{3} \mathrm{~K}\right)$ as projectile and "cold" molecules, kept at liquid air temperature $\left(T_{\text {rot }} \simeq 10^{2} \mathrm{~K}\right.$ ), as target. At low velocity the measured cross-sections mainly probe the spherical component of the interaction, since the collisional time is larger than the period of a molecular rotations-the critical time needed to induce an angular averaging of the interaction. As the collisional velocity increases the anisotropy enters into play, since the collisions tends to assume a "sudden" character. The novel technique developed in our group, first reported in Nature in 1994 [20], for cooling oxygen to the lowest vibrorotational state and for aligning the rotational angular momentum, allows the control of the relative orientation of the colliding molecules and provides more direct information on the interaction anisotropy from the measurement of changes in the smooth component, in the amplitude and in the extrema position in the "glory" pattern, as a function of the projectile molecular alignment degree.

Along these lines, a simultaneous analysis of our experimental cross-sections and of available second virial coefficients has been carried out to obtain a reliable interaction for dimers of the major components of the atmosphere. Scattering experiments with "hot" projectile molecules had been also performed on the $\mathrm{N}_{2}-\mathrm{N}_{2}$ and $\mathrm{O}_{2}-\mathrm{N}_{2}$ systems. These previous results on the $\mathrm{N}_{2}-\mathrm{N}_{2}$ system [8] have been reanalyzed, and a characterization of the $\mathrm{N}_{2}-\mathrm{O}_{2}$ complex has been presented in a recent paper [5]. These results indicate that most of the bonding in the dimers comes from van der Waals (repulsion + dispersion) and electrostatic (permanent quadrupole-permanent quadrupole) forces. On the other hand, chemical (spin-spin) contributions are not negligible for $\left(\mathrm{O}_{2}\right)_{2}$, which is an open-shell-openshell system [1]. Therefore the geometrical properties of the three dimers has been found to show interesting differences, to be seen in the next section.

\section{POTENTIAL ENERGY SURFACES}

We find useful to represent the interaction potential for a dimer of homonuclear diatomic molecules [1, 10, 21] as a spherical harmonic expansion, separating radial and angular dependencies

$$
V\left(R, \theta_{a}, \theta_{b}, \phi\right)=4 \pi \sum_{l_{a} l_{b} l} \mathrm{~V}_{l_{a} l_{b} l}(R) \mathcal{y}_{l_{a} l_{b} l}\left(\theta_{a}, \theta_{b}, \phi\right)
$$

where $\mathcal{Y}_{l_{a} l_{b} l}\left(\theta_{a}, \theta_{b}, \phi\right)$ are bipolar spherical harmonics (angles are as in Figure 1). The radial coefficients $\mathrm{V}_{l_{a} l_{b} l}(R)$ include different types of contributions to the interaction potential (electrostatic, dispersion, repulsion due to overlap, induction, spin-spin coupling, ...). For the three system, we look for a compact expansion,
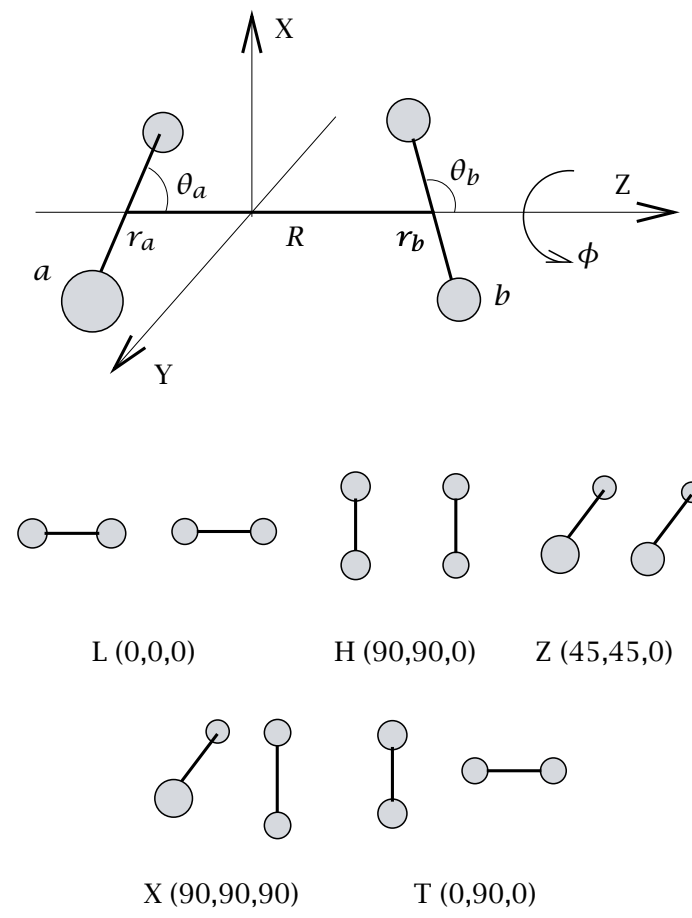

Figure 1. Characteristic configurations [angular coordinates are $\left(\theta_{a}, \theta_{b}, \phi\right)$, where $\phi$ is $\left.\phi_{a}-\phi_{b}\right]$. 
truncating the series to a small number of physically motivated terms. The number of terms used are six for the $\mathrm{N}_{2}-\mathrm{O}_{2}$ system corresponding to the number of configurations of the dimer (see Figure 1: for $\mathrm{N}_{2}-\mathrm{N}_{2}$ and $\mathrm{O}_{2}-\mathrm{O}_{2}$ this number of terms is reduced at five and four respectively, see below).

The radial coefficients are derived from the analysis of experimental data assisted by empirical correlation formulas [22]. The $\mathrm{V}_{000}$ term has the meaning of a typical isotropic van der Waals interaction, arising from a short-range repulsion, associated to the spherical "size" of both partners, and a long-range dispersion attraction, depending on the spherical polarizability of each of the two monomers. The $\mathrm{V}_{202}$ and $\mathrm{V}_{022}$ coefficients are associated to the orientational anisotropy of each diatom when the other one is considered as a spherical partner: this is the anisotropy expected in the diatom-"pseudoatom" limits. For $\mathrm{N}_{2}-\mathrm{N}_{2}$ and $\mathrm{O}_{2}-\mathrm{O}_{2}$ these terms are equal but not in the case of $\mathrm{N}_{2}-\mathrm{O}_{2}$ where two different limits are possible. To be specific, in the analysis of the scattering of "hot" $\mathrm{O}_{2}\left(10^{3} \mathrm{~K}\right)$ by "cold" $\mathrm{N}_{2}\left(10^{2} \mathrm{~K}\right), \mathrm{O}_{2}$ acts as a "pseudoatom", and therefore cross-sections at high velocity provide information on the potential in this limiting case. $\mathrm{V}_{220}$ and $\mathrm{V}_{222}$ are coefficients which introduce corrections to the repulsion arising from the mutual orientation of both molecules. $\mathrm{V}_{224}$ is an electrostatic component exclusively depending on the quadrupole-quadrupole interaction, which can be accurately estimated. [This term is negligible in the $\mathrm{O}_{2}-\mathrm{O}_{2}$ system]. Induction contributions due to permanent quadrupole-induced multipole interactions, which should be included in the $\mathrm{V}_{202}, \mathrm{~V}_{022}, \mathrm{~V}_{220}$, and $\mathrm{V}_{222}$ coefficients, play only a minor role especially in the intermediate region of the intermolecular distance $R$, which are those of interest here.

The analysis of the total cross-section measured as a function of the collision velocity provides direct information on the $\mathrm{V}_{000}$ component on the low velocity range, and yields information on the strength of the leading anisotropic terms from the "glory" quenching observed at high velocity, which is an attenuation on the amplitude of an interference phenomenon which shows up in the molecular beam scattering. The second virial coefficient is affected by all the radial terms in the low temperature range while in the high temperature limit it mainly depends on the spherical component.

The intermolecular potentials in the limiting configurations of the dimers are shown in Figure 2, while relevant features of the interactions are compared in Table 1. For the $\mathrm{O}_{2}-\mathrm{O}_{2}$ dimer the additional contribution due to the spin-spin coupling, for which information had been obtained from the analysis of the "glory" quenching in the low velocity range [1], had been included in the expansion and yielded the splittings among singlet, triplet and quintet surfaces.

Interestingly, the three dimers differ both for their geometry and for the nature of the bond. For $\mathrm{N}_{2}-\mathrm{N}_{2}$ (no
Table 1. Equilibrium distances $R_{m}$ (in $\AA$ ) and well depth energies $E$ (in $\mathrm{cm}^{-1} ; 1 \mathrm{~cm}^{-1}=0.1240 \mathrm{meV}$ ) for selected geometries and for the spherically average $\mathrm{V}_{000}$. Uncertainties are estimated as $\pm 6 \mathrm{~cm}^{-1}$ for $E$ and $\pm 0.07 \AA$ for $R_{m}$. In Italics the most stable configurations.

\begin{tabular}{ccccccc}
\hline System & \multicolumn{2}{c}{$V_{\mathrm{N}_{2}-\mathrm{N}_{2}}$} & \multicolumn{2}{c}{$V_{\mathrm{O}_{2}-\mathrm{O}_{2}}{ }^{\mathrm{a}}$} & \multicolumn{2}{c}{$V_{\mathrm{N}_{2}-\mathrm{O}_{2}}$} \\
\hline configuration & $E$ & $R_{m}$ & $E$ & $R_{m}$ & $E$ & $R_{m}$ \\
$\mathbf{H}$ & 95 & 3.81 & 137 & 3.56 & 120 & 3.70 \\
$\mathbf{X}$ & 97 & 3.87 & 123 & 3.63 & 130 & 3.66 \\
$\mathbf{T}$ & 107 & 4.03 & 129 & 3.74 & $100^{\mathrm{b}}$ & $4.00^{\mathrm{b}}$ \\
$\mathbf{Z}$ & 74 & 4.30 & 77 & 4.03 & 71 & 4.24 \\
$\mathbf{L}$ & 30 & 4.65 & 73 & 4.26 & 53 & 4.47 \\
$\mathrm{~V}_{000}$ & 79 & 4.11 & 107 & 3.81 & 88 & 4.02 \\
\hline
\end{tabular}

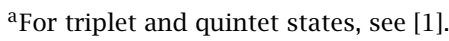

${ }^{\mathrm{b}}$ Average of the two $\mathrm{T}$ configurations.

electronic spin) the basic feature which determines the equilibrium geometry is the quadrupole-quadrupole interaction, which favors the T-configuration (see Figure 2). In the case of oxygen the equilibrium geometry obtained for the ground singlet state is the H-configuration (see Figure 2). A crucial role is played by the spin-spin interaction, in spite that its contribution to the bond is small (approximately 15\% at the equilibrium configuration [1]). This leads to the $\mathbf{H}$ geometry as the most stable configuration, where the two $\mathrm{O}_{2}$ molecules are parallel. Indeed, the binding forces are slightly stronger than in the nitrogen case because of such contribution from the spin-spin interaction. Finally, a $\mathbf{X}$-configuration (see Figure 2) is found to be the most stable for $\mathrm{N}_{2}-\mathrm{O}_{2}$ : here no role is played by spin interaction and the quadrupole-quadrupole interaction is not strong enough to stabilize the two possible T-configurations. These potential energy surfaces are considered to be such that their handling and the physical interpretation of their terms make them "realistic" in the sense that they reproduce micro and macroscopic quantities experimentally available. Since spectroscopic information, when available, has not been fully analyzed (see e.g. [23]), and more spectra may be taken in the future, whose analysis will require theoretical guidance, we proceeded to study the associated quantum dynamics.

\section{CLUSTER DYNAMICS: BOUND STATES AND SPECTRAL FEATURES}

A particular effort has been addressed to the study of the dynamics within the dimer and to the characterization of the low lying rotovibrational states in view of potential interest for the analysis of spectral features in atmospheric research. Calculations of the bound rovibrational states of the dimers have been performed for $J \leq 6$ by solving the secular problem over the exact Hamiltonian. We have calculated the rotovibrational 


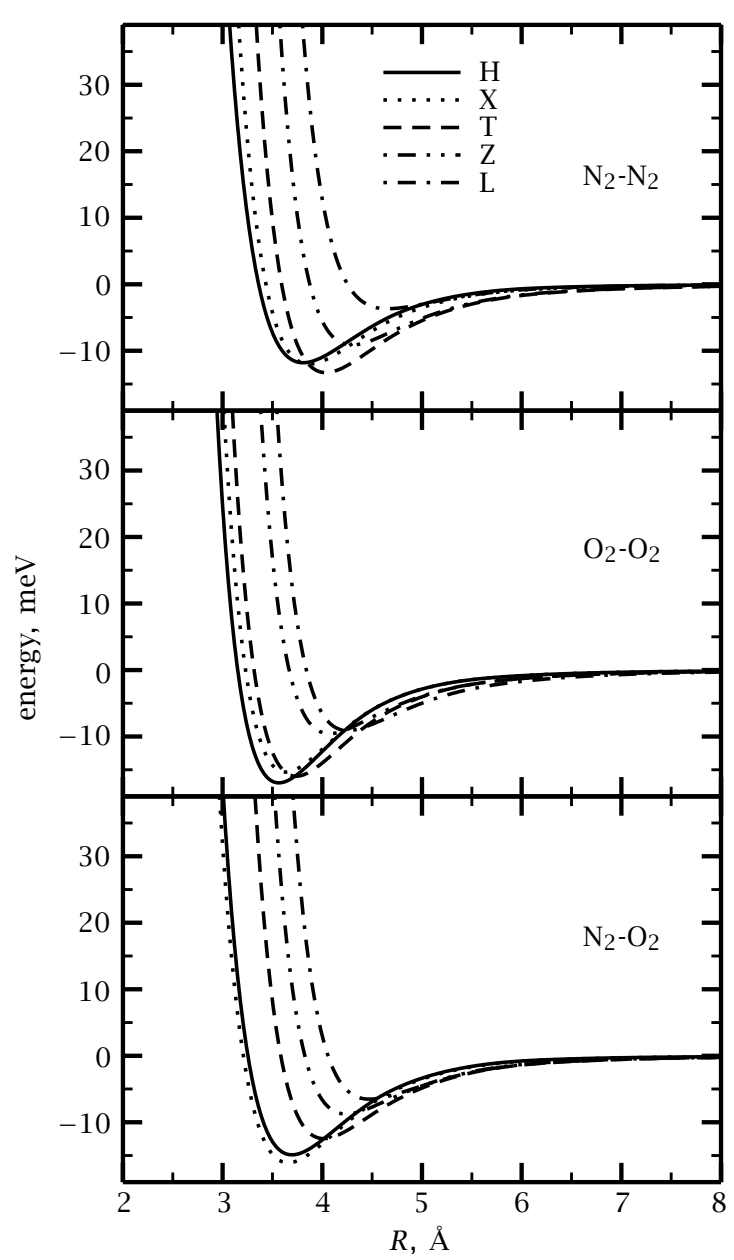

Figure 2. Cuts of potential energy surfaces $\left(1 \AA=10^{-8} \mathrm{~cm}\right)$ corresponding to the configurations of Figure 1. The $\mathrm{O}_{2}$ $\mathrm{O}_{2}$ curves are for the singlet surface (for the triplet and quintet cases see ref. [1]); for the $\mathrm{N}_{2}-\mathrm{O}_{2}$ the two $\mathrm{T}$ curves are indistinguishable on this drawing.

levels for the new surfaces for the dimers $\mathrm{N}_{2}-\mathrm{N}_{2}, \mathrm{~N}_{2}-\mathrm{O}_{2}$ and for $\mathrm{O}_{2}-\mathrm{O}_{2}$ (for all three surfaces, singlet, triplet, quintet) [1]. A summary of results and their discussion follows. Full account of all available data has been given in $[2,4,5]$.

Our potentials enable us to computing rovibrational energy levels of the dimers treating each monomer $\mathrm{O}_{2}$, $\mathrm{N}_{2}$ as a rigid rotor, decoupling intermonomer (van der Waals) and intramonomer vibrations (by almost two orders of magnitude larger).

The theory to solve the Schrödinger equation for this Hamiltonian is reported in ref. [21]. The full quantum mechanical calculations of bound states are carried out using the program BOUND [24], where the intermolecular distance $R$ is treated as a scattering coordinate, and a basis set expansion is used for the remaining degrees of freedom. The coupled equations are then solved using the standard techniques of scattering theory, but with bound state boundary conditions. This method is found to be particularly appropriate for van der Waals complexes, where there is wideamplitude vibrational motion along the intermolecular coordinate [25]. Previously, the $\mathrm{N}_{2}-\mathrm{N}_{2}$ and $\mathrm{O}_{2}-\mathrm{O}_{2}$ dimers had been studied in the body-fixed formulation [9, 26], which has some advantages when one neglects Coriolis couplings, an approximation that we do not make but assess below.

It is found that some of the levels which are degenerate without Coriolis coupling are actually split. Because of Coriolis mixing, there are energy levels (with $J>0$ but different $K$ ) which perturb each other leading to a stabilization of the order of $1 \mathrm{~cm}^{-1}$ in some cases. The splitting is of the order of $0.03 \mathrm{~cm}^{-1}$ for the $J=1$ case in some levels, but for higher $J$ can be more than 20 times larger. Computing time for the exact close coupling calculation is only twice than for the helicity decoupling approximation for $J=1$, but becomes quite demanding as $J$ increases. For example, for $J=6$ it is 40 times larger.

We calculated also other spectral aspects, vibrational frequencies, rotational constants, etc. The results can be compared with previous works $[9,26]$ where the potential surfaces used are very different: we found that although some features are similar, zero point and dissociation energies differ (Table 2 should be compared with corresponding ones in refs. [9, 26]).

Surprisingly we have seen that although the used potential energy surfaces can have different topography, comparable results are obtained for some spectroscopic observables, in spite of the fact that geometry and values of well depths and positions differ significantly, what varies is their ability to reproduce experimental data (integral cross-sections and second virial coefficient).

We also calculated harmonic frequencies through the second derivative of the potential around the equilibrium geometry of the dimers given in Table 2. From these values we obtain the energy levels of each vibrational level (harmonic), and the values calculated are also very different from the exact results, because the interaction forces are very weak and the potential anisotropy is important.

We can conclude that the range and strength in the bonds of the three dimers $\mathrm{N}_{2}-\mathrm{N}_{2}, \mathrm{O}_{2}-\mathrm{O}_{2}$, and $\mathrm{N}_{2}-\mathrm{O}_{2}$ present characteristics more of those of clusters than of weakly bound molecules and that the interaction between the monomers is very anharmonic.

In this work we have dedicated special emphasis to the characterization of the low lying energetic levels and of the associated wavefunctions exploiting the availability of realistic potential energy surfaces. This study leads to a faithful representation of internal dynamics of the system involving levels pertaining to the lowest energy states for the dimers. 
Table 2. Selected vibrational frequencies [harmonic: calculated from second derivatives; exact: close-coupling calculations]. (All values are in $\mathrm{cm}^{-1}$ ).

\begin{tabular}{cccccc}
\hline & & $\omega_{R}$ & $\omega_{\left(\phi_{b}-\phi_{a}\right)}$ & Zero point energy & Binding energy \\
\hline \multirow{3}{*}{$\mathrm{N}_{2}-\mathrm{N}_{2}$} & exact $^{\mathrm{a}}$ & 30.78 & 2.47 & 27.27 & 79.87 \\
& harmonic $^{\mathrm{a}}$ & 28.70 & 12.77 & 35.73 & 71.40 \\
& exact $^{\mathrm{b}}$ & 33.2 & 8.1 & 47.1 & 74.94 \\
& harmonic $^{\mathrm{b}}$ & 39.2 & 13.9 & 48.7 & 73.3 \\
$\mathrm{O}_{2}-\mathrm{O}_{2}$ & exact $^{\mathrm{a}}$ & 29.26 & 13.45 & 22.59 & 120.26 \\
& harmonic $^{\mathrm{a}}$ & 48.28 & 11.32 & 65.97 & 71.15 \\
& exact $^{\mathrm{c}}$ & 23.59 & 6.92 & 79.82 & 73.18 \\
$\mathrm{~N}_{2}-\mathrm{O}_{2}$ & harmonic $^{\mathrm{c}}$ & 43 & 18 & 78 & 75 \\
& exact $^{\mathrm{a}}$ & 28.5 & 9.7 & 12.0 & 120.6 \\
\hline
\end{tabular}

apresent work;

${ }^{\mathrm{b}}$ Ref. [9];

${ }^{\mathrm{c}}$ Ref. [26].
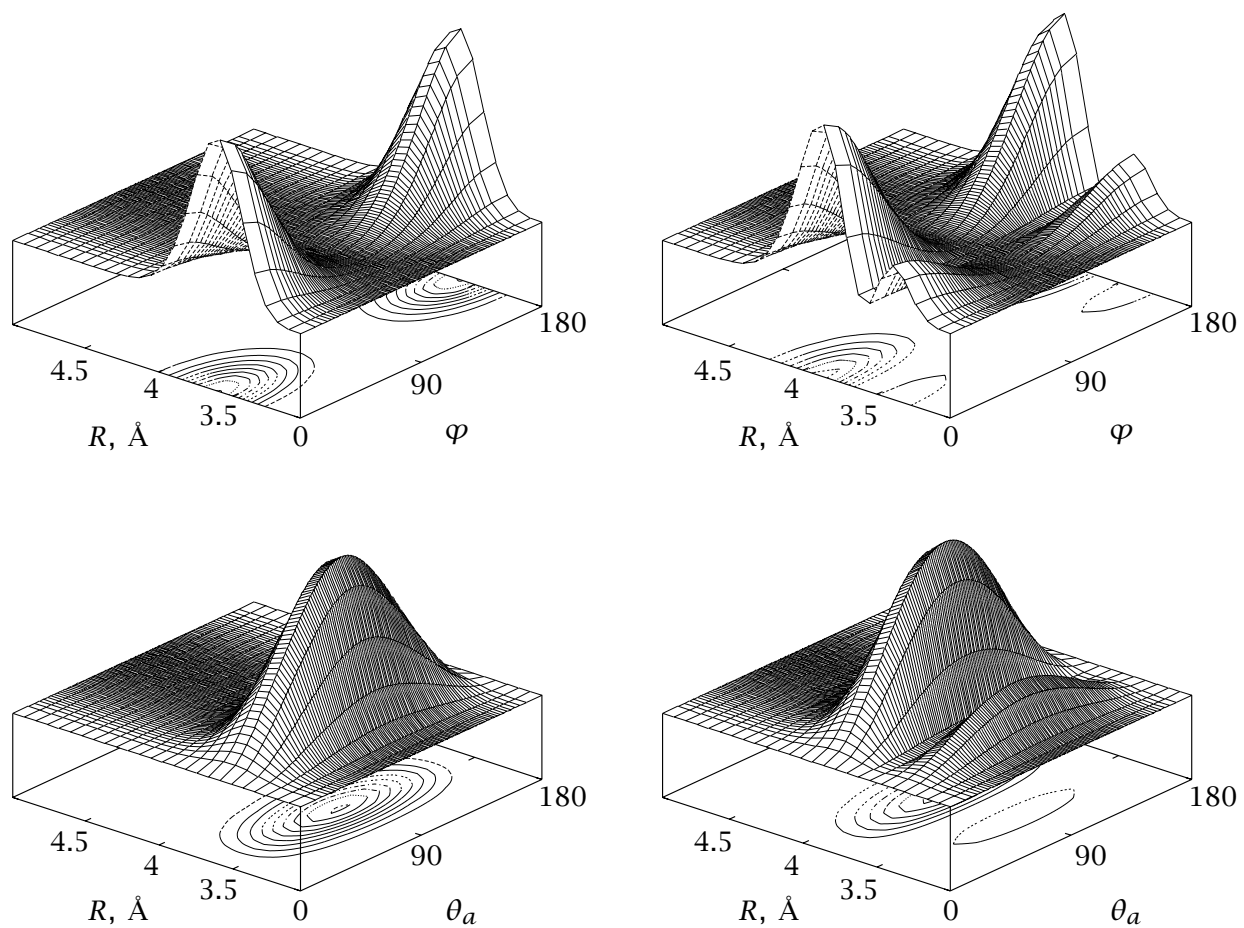

Figure 3. Views of the square modulus of the wavefunctions for the $\mathrm{O}_{2}-\mathrm{O}_{2}$ system. Right: the lowest $J=0 A_{1}^{+}$state; Left: the second $J=O A_{1}^{+}$excited state. In all plots $\theta_{b}=90^{\circ}$. In the upper plots $\theta_{a}$ is fixed at $90^{\circ}$ and in the lower plots $\phi$ is fixed at $0^{\circ}$.

To obtain a complete characterization of the vibrational states and of related motions within the dimer a simultaneous analysis of the radial and angular behavior of the total wavefunction is required. The program BOUND provides the rotovibrational energy levels but not the associated wavefunctions. In order to obtain the latter we make recourse to a method developed by Thornley and Hutson [27]. Specifically, starting from a known eigenvalue of the wavefunction at a particular value of the $R$ coordinate we can recover the behavior of the involved wavefunction in a wide $R$ range making use of the equations for the propagation of the logarithmic derivative. This mechanism allows us to examine the total wavefunction, facilitating the assignment of the fundamental vibrations within the dimers.

Distributions of such probabilities for the ground and second excited state, represented in terms of the coordinates $\phi=\phi_{a}-\phi_{b}$ and $\theta_{a}$ are shown in Figure 3 . 
The plots in this figure suggest a $\mathbf{H}$ shape conformation for the ground state complex: the maximum probability occurs at $\theta_{a}=90^{\circ}$ and $\phi=0^{\circ}$ and $180^{\circ}$. In the second excited state (Figure 3 left panel), the wavefunction presents a maximum probability also for the $\mathbf{H}$ conformation. Nodes in the angular part are absent and therefore this level exhibits features close to the ground state. However a node in the radial part can be recognized and assigned to an excitation in the stretching mode, characterized by a marked anharmonic behavior. It can be seen in fact that a poor symmetric distribution of probability in the $R$ coordinate, and most of probability is confined at the larger value of $R$.

The results are not presented here for brevity, but they are accessible through the net http://bong.chm. unipg.it.

\section{CONCLUSIONS AND FURTHER EXTENSIONS}

Implications for the interpretation of recent laboratory [23] and atmospheric [28] spectroscopic observations, as well as of current measurements of high pressure behavior of oxygen [29] are amenable to be discussed in this framework. This work provides the ground for the interpretation of complicated band features in rotational spectral, as exemplified by the case of oxygen. Still appears to be valid the statement [30] that spectral analysis alone is not sufficient to extract information on structure and bonding, and the combined use of scattering and gaseous properties information is therefore confirmed to be crucial.

Progress along the lines outlined above is accounted for in [31]. In the context of atmospheric photochemistry and physics, and climate (Figure 4) probably the most important is the interaction of $\mathrm{H}_{2} \mathrm{O}$ with $\mathrm{N}_{2}, \mathrm{O}_{2}$, and itself giving rise to a variety of processes that have received little attention and are not well understood.

Among these are homogeneous nucleation of water, water cluster mediated photochemical reactions, and the radiative properties of water vapor and water clusters in the visible/near IR and mid-IR spectral ranges. There has been a long-standing controversy in atmospheric physics as to the origin of the infrared continuum absortin by water vapour and of the potential role for dimers in explaining this continuum absortion. Another recent challenge concerns our quantitative understanding of the atmospheric absortion in clear skies, in which water clusters may play an important role. Following recent reports of the first spectroscopic detection of water dimers $\left(\mathrm{H}_{2} \mathrm{O}\right)_{2}$ in the Earth's atmosphere [32] and the mutual role water dimers can play in atmospheric processes, the present research may contribute to conduct in-depth studies of the physics of weakly interacting molecules and their impact for atmospheric physics and climate.

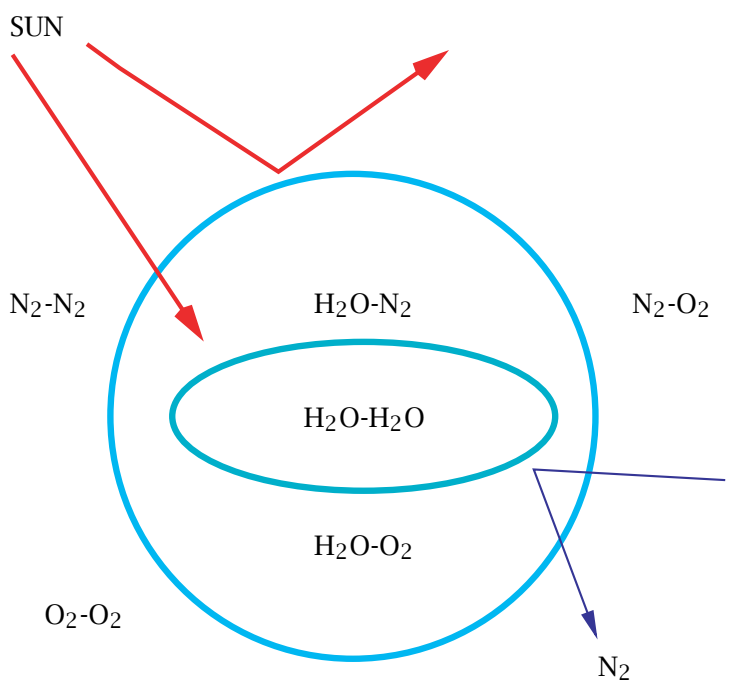

Figure 4. Schematic view of currently investigated systems of relevance for atmospheric photochemistry and physics.

To give a flavour of the accuracy of our technique in the case of water molecules, we have reported in [31] the test of a recent ab-initio potential for the $\mathrm{H}_{2} \mathrm{O}$ He dimer [33] on the measured cross section integral cross section data. Since in our experimental conditions water molecules in the beam are kept at $500 \mathrm{~K}$, the molecules rotate so fast to fully average the interaction during the cross sections collision and we calculated the cross sections with the interaction potential properly averaged over all orientations in space. Further work on interactions of $\mathrm{H}_{2} \mathrm{O}$ with the rare gases and with those molecules relevant for atmospheric studies (especially $\mathrm{Ar}, \mathrm{N}_{2}, \mathrm{O}_{2}, \mathrm{CO}_{2}, \ldots$ ) is currently being completed.

\section{ACKNOWLEDGMENTS}

This work is supported by the Italian MIUR, INFM, ASI and ENEA, and by European Union contracts.

\section{REFERENCES}

[1] V. Aquilanti, D. Ascenzi, M. Bartolomei, D. Cappelletti, S. Cavalli, M. De Castro Vitores, and F. Pirani, Phys. Rev. Lett. 82 (1999), 69; J. Am. Chem. Soc. 121 (1999), 10794.

[2] V. Aquilanti, M. Bartolomei, D. Cappelletti, E. Carmona-Novillo, and F. Pirani, J. Chem. Phys. 117 (2002), 615.

[3] V. Aquilanti, M. Bartolomei, D. Cappelletti, E. Carmona-Novillo, and F. Pirani, Phys. Chem. Chem. Phys. 3 (2001), 3891. 
[4] V. Aquilanti, E. Carmona-Novillo, and F. Pirani, Phys. Chem. Chem. Phys. 4 (2002), 4970.

[5] V. Aquilanti, M. Bartolomei, E. Carmona-Novillo, and F. Pirani, J. Chem. Phys. 118 (2003), 2214.

[6] E. Carmona-Novillo, F. Pirani, and V. Aquilanti, Int. J. Quantum Chem. (2003), in press.

[7] C. A. Long, G. Henderson, and G. E. Ewing, Chem. Phys. 2 (1973), 485.

[8] D. Cappelletti, F. Vecchiocattivi, F. Pirani, E. L. Heck, and A. S. Dickinson, Mol. Phys. 93 (1998), 485.

[9] J. Tennyson, A. van der Avoird, J. Chem. Phys. 77 (1982), 5664.

[10] M. C. van Hemert, P. E. S. Wormer, and A. van der Avoird, Phys. Rev. Lett. 51 (1993), 1167.

[11] O. Couronne and Y. Ellinger, Chem. Phys. Lett. 306 (1999), 71.

[12] A. Wada, H. Kanamori, and S. Iwata, J. Chem. Phys. 109 (1998), 9434.

[13] A. H. Hamdani, A. Shen, Y. Dong, H. Gao, and Z. Ma, Chem. Phys. Lett. 325 (2000), 610.

[14] F. Uhlik, Z. Slanina, and A. Hinchliffe, J. Mol. Struct. (Theochem) 282 (1993), 271.

[15] J. R. Stallcop and H. Partridge, Chem. Phys. Lett. 281 (1997), 212.

[16] Z. Slanina, F. Uhlik, and W. B. De Almeida, Thermochimica Acta 231 (1994), 55.

[17] G. E. Ewing and J. P. M. Trusler, Physica A 184 (1992), 415.

[18] A. J. Brewer and J. W. Vaughn, J. Chem. Phys. 50 (1969), 2960.

[19] K. R. Hall and G. A. Iglesias-Silva, J. Chem. Eng. Data 39 (1994), 873.

[20] V. Aquilanti, D. Ascenzi, D. Cappelletti, and F. Pirani, Nature (London) 371 (1994), 399.

[21] S. Green, J. Chem. Phys. 62 (1975), 2271.
[22] F. Pirani, D. Cappelletti, and G. Liuti, Chem. Phys. Lett. 350 (2001), 286.

[23] A. Campargue, L. Biennier, A. Kachanov, R. Jost, B. Bussery-Honvault, V. Veyret, S. Churassy, and R. Bacis, Chem. Phys. Lett. 288 (1998), 734;

L. Biennier, D. Romanini, A. Kachanov, A. Campargue, B. Bussery-Honvault, and R. Bacis, J. Chem. Phys. 14 (2000), 6309.

[24] J. M. Hutson, BOUND, Computer Code, Version 5 (1993), distributed by Collaborative Computational Project no. 6 of the Science and Engeneering Research Council, UK.

[25] J. M. Hutson, Comp. Phys. Comm. 84 (1994), 1.

[26] B. Bussery-Honvault and V. Veyret, Phys. Chem. Chem. Phys. 1 (1999), 3387.

[27] A. E. Thornley and J. M. Hutson, J. Chem. Phys. 101 (1994), 5578.

[28] K. Pfeilsticker, H. Bösch, C. Camy-Peyret, R. Fitzenberger, H. Harder, and H. Osterkamp, Geophys. Res. Lett. 28 (2001), 4595.

[29] F. A. Gorelli, L. Ulivi, M. Santoro, and R. Bini, Phys. Rev. Lett. 83 (1999), 4093.

[30] C. A. Long and G. E. Ewing, Chem. Phys. Lett. 9 (1971), 225; J. Chem. Phys. 58 (1973), 4824; Acc. Chem. Res. 8 (1975), 185.

[31] V. Aquilanti, M. Bartolomei, D. Cappelletti, E. Carmona-Novillo, E. Cornicchi, M. Moix-Teixidor, M. Sabidó, and F. Pirani, Weakly Interacting Molecular Pairs: Unconventional Absorbers of Radiation in the Atmosphere, (C. Camy-Peyret and A. Vigasin, Eds.), Kluwer, Dordrecht, 2003, pp. 169-182.

[32] K. Pfeilsticker, A. Lotters, C. Peters, and H. Bösch, Science 300 (2003), 2078.

[33] M. P. Hodges, R. J. Wheatley, and A. H. Harvey, J. Chem. Phys. 116 (2002), 1397. 


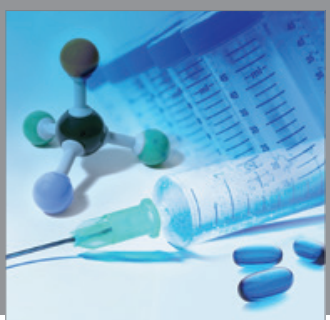

International Journal of

Medicinal Chemistry

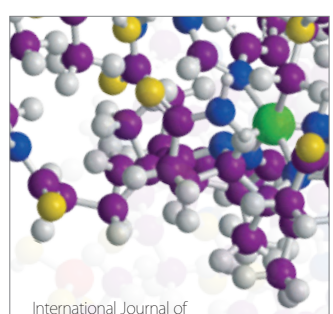

Carbohydrate Chemistry

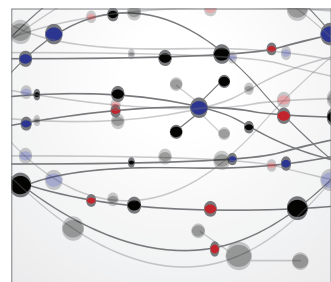

The Scientific World Journal
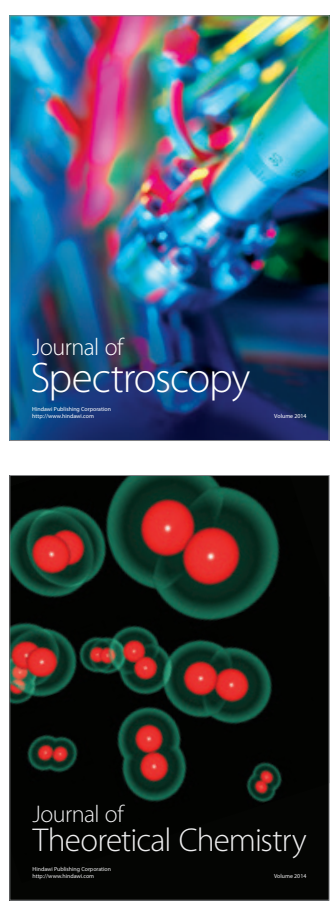
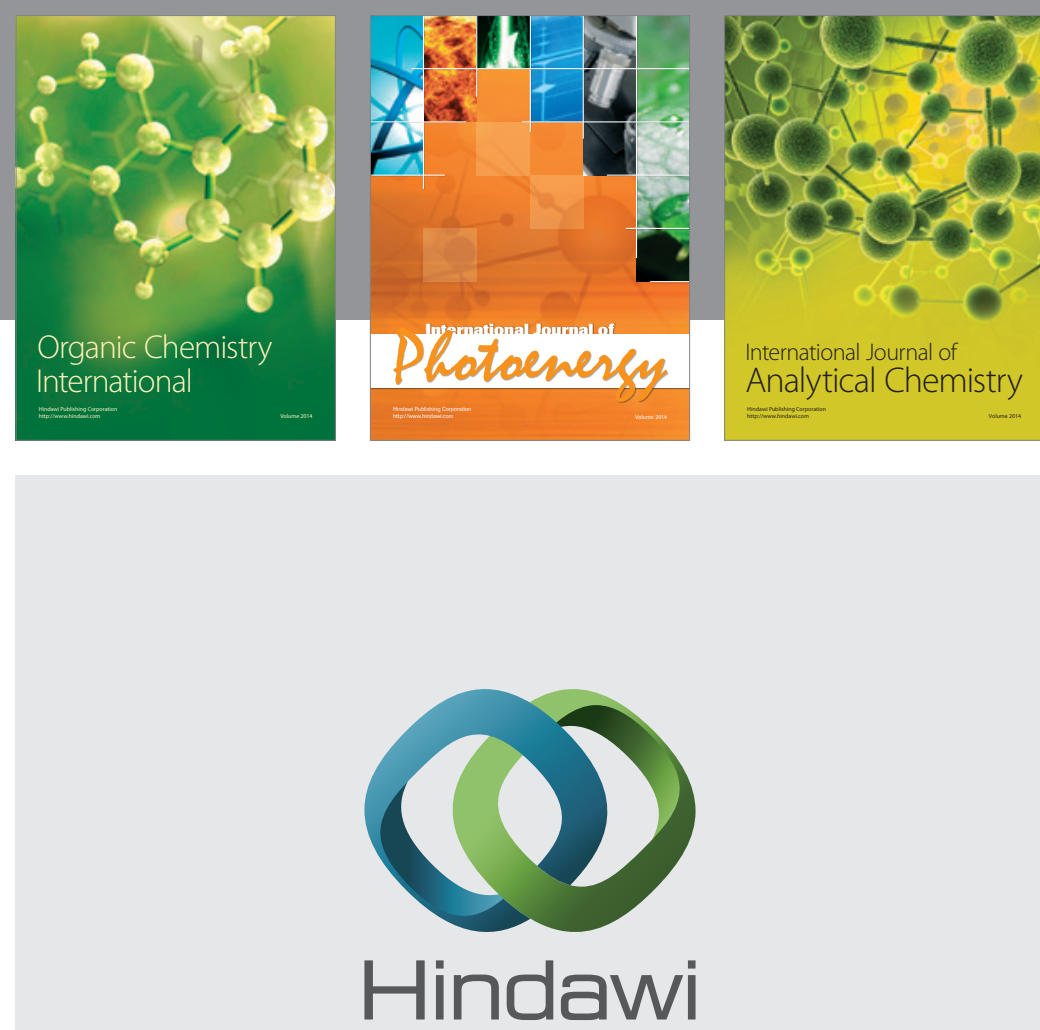

Submit your manuscripts at

http://www.hindawi.com
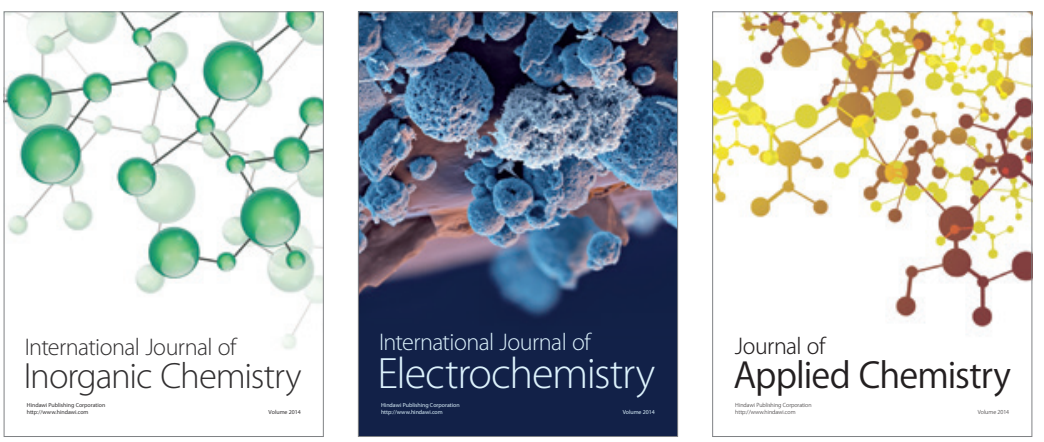

Journal of

Applied Chemistry
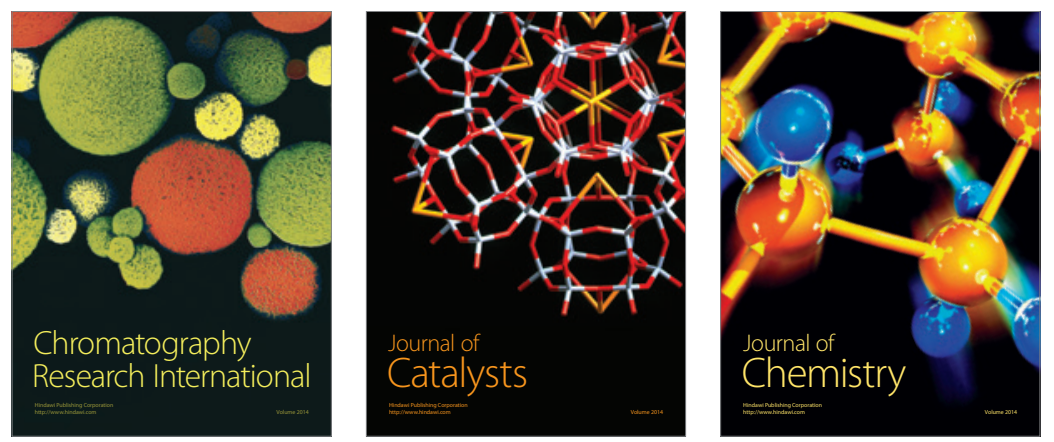
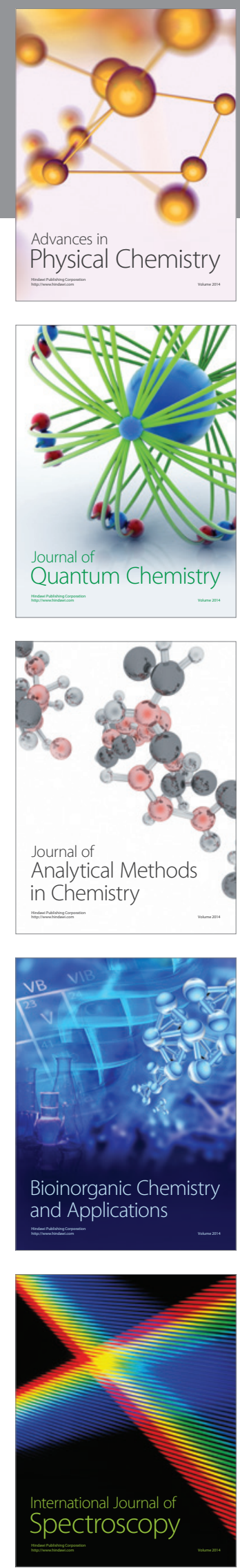